\title{
Coronary atherosclerosis as the main endpoint of non-invasive imaging in cardiology: a narrative review
}

\author{
Erica Maffei ${ }^{1}$, Bruna Punzo ${ }^{2}$, Carlo Cavaliere ${ }^{2}$, Eduardo Bossone $^{3}$, Luca Saba $^{4}$, Filippo Cademartiri $^{2}$ \\ ${ }^{1}$ Department of Radiology, Area Vasta 1, ASUR Marche, Urbino (PU), Italy; ${ }^{2}$ Department of Radiology, SDN IRCCS, Naples, Italy; ${ }^{3}$ Department of \\ Cardiology, Ospedale Cardarelli, Naples, Italy; ${ }^{4}$ Department of Radiology, University of Cagliari, Italy \\ Contributions: (I) Conception and design: E Maffei, F Cademartiri; (II) Administrative support: None; (III) Provision of study materials or patients: \\ None; (IV) Collection and assembly of data: All authors; (V) Data analysis and interpretation: E Maffei, B Punzo, F Cademartiri; (VI) Manuscript \\ writing: All authors; (VII) Final approval of manuscript: All authors. \\ Correspondence to: Prof. Dr. Filippo Cademartiri, MD, PhD. Chairman, Department of Radiology, Area Vasta 1, ASUR Marche, Ospedale Civile "Santa \\ Maria della Misericordia” di Urbino, viale Federico Comandino, 70-61029 Urbino (PU), Italy. Email: filippocademartiri@gmail.com.
}

\begin{abstract}
The change of paradigm determined by the introduction of cardiac computed tomography (CCT) in the field of cardiovascular medicine has allowed new evidence to emerge. These evidences point towards a major role, probably the most important one in terms of prognostic impact, in the detection, characterization and quantification of atherosclerosis as the main driver and endpoint for the management of coronary artery disease (CAD). Extensive literature has been published in the last decade with large numbers and patients' populations, investigating several aspects and correlations between atherosclerotic plaque features and risk factors; also, the relationship between plaque features, both with qualitative and quantitative approaches, and cardiovascular events has been investigated. More recent studies have also pointed out the relationship between the knowledge and classification of sub-clinical atherosclerosis and the induced modification of medical therapy (both aggressiveness and compliance) that is most likely able to increase the effect of antiatherosclerotic drugs, hence significantly improving prognosis. Non-invasive assessment of CAD by means of CCT is becoming the primary tool for management and also the most important parameter for the comprehension of natural history of CAD and how the therapies we adopt are affecting plaque burden as a whole. In this review we will address the modern concepts of CAD driven understanding and management of cardiovascular disease.
\end{abstract}

Keywords: Coronary artery disease (CAD); cardiac computed tomography (CCT); atherosclerosis; diagnosis; prognosis; therapy; optimal medical therapy (OMT)

Submitted May 22, 2020. Accepted for publication Aug 07, 2020.

doi: $10.21037 / \mathrm{cdt}-20-525$

View this article at: http://dx.doi.org/10.21037/cdt-20-525

\section{Introduction}

Imaging in cardiology has been the backbone of clinical decision making (1-24) and also one of the major drivers in clinical research (25-32). There is no doubt that many important steps have been made in clinical cardiology through the application of standard imaging modalities (e.g., echocardiography, SPECT, CMR, Invasive Angiography with all additional intravascular tools). However, until the introduction of cardiac computed tomography (CCT), there have always been a dichotomy between non-invasive and invasive techniques; they were complementary and most of all coronary imaging was only possible with invasive techniques.

In the early years of this millennium CCT was developed and progressively put in place; over 2 decades it has become a robust clinical method with a very wide spectrum of clinical application and its clinical role is expanding every year. CCT's role has been properly recognized in guidelines (21) after some major trials (29-32) and spans from diagnosis, to 



Figure 1 CCT of different CAD phenotypes. The figure shows different CAD phenotypes. (A) A normal LAD straightened with axial section of the vessel. (B) A patient with non-obstructive phenotype characterized by a mixed quality of disease (i.e., concomitant presence of calcified and non-calcified atherosclerosis). (C) A patient with severe CAD and obstructive phenotype on proximal-mid LAD and ostial D1 which entails a heavy burden of calcified disease. LAD, left anterior descending coronary artery; D1, the 1st diagonal branch; CCT, cardiac computed tomography; CAD, coronary artery disease.

prognosis, to surveillance, to atherosclerosis phenotyping, to pre-interventional planning (coronary and structural). CCT has become the real missing piece of the puzzle to complete the armamentarium of non-invasive techniques in cardiology (Figure 1).

What we as experienced operator struggle with every day is somehow to communicate that the previous paradigm of coronary artery disease (CAD) approach is not there anymore. Cardiovascular medicine has developed and underwent a major change driven by technology that today it is not acceptable anymore to keep thinking in terms of an ischemia-centric clinical environment. Prevalence of CAD is increasing but morbidity and mortality are slightly reducing, the "face" (i.e., the phenotype) of atherosclerosis is changing because of the changes in our lifestyle, because of the chronic therapies we assume, because of other factors we are not completely able to understand. And in addition to this there is a very simple concept to really fix in our mind: "ischemia is not a disease; atherosclerosis is the disease"; hence, performing early coronary imaging we get much more than one step closer to the disease we want to treat and prevent.

We present the following article in accordance with the Narrative Review reporting checklist (available at http:// dx.doi.org/10.21037/cdt-20-525).

\section{The history leading to the "New Paradigm" (Figure 2)}

In several fields of medicine when we talk and deal with a disease using tests and imaging methods, we tend to focus more and more on direct signs of the primary diseases itself. Modern medicine still starts from signs \& symptoms collected while talking and examining the patient, but as 


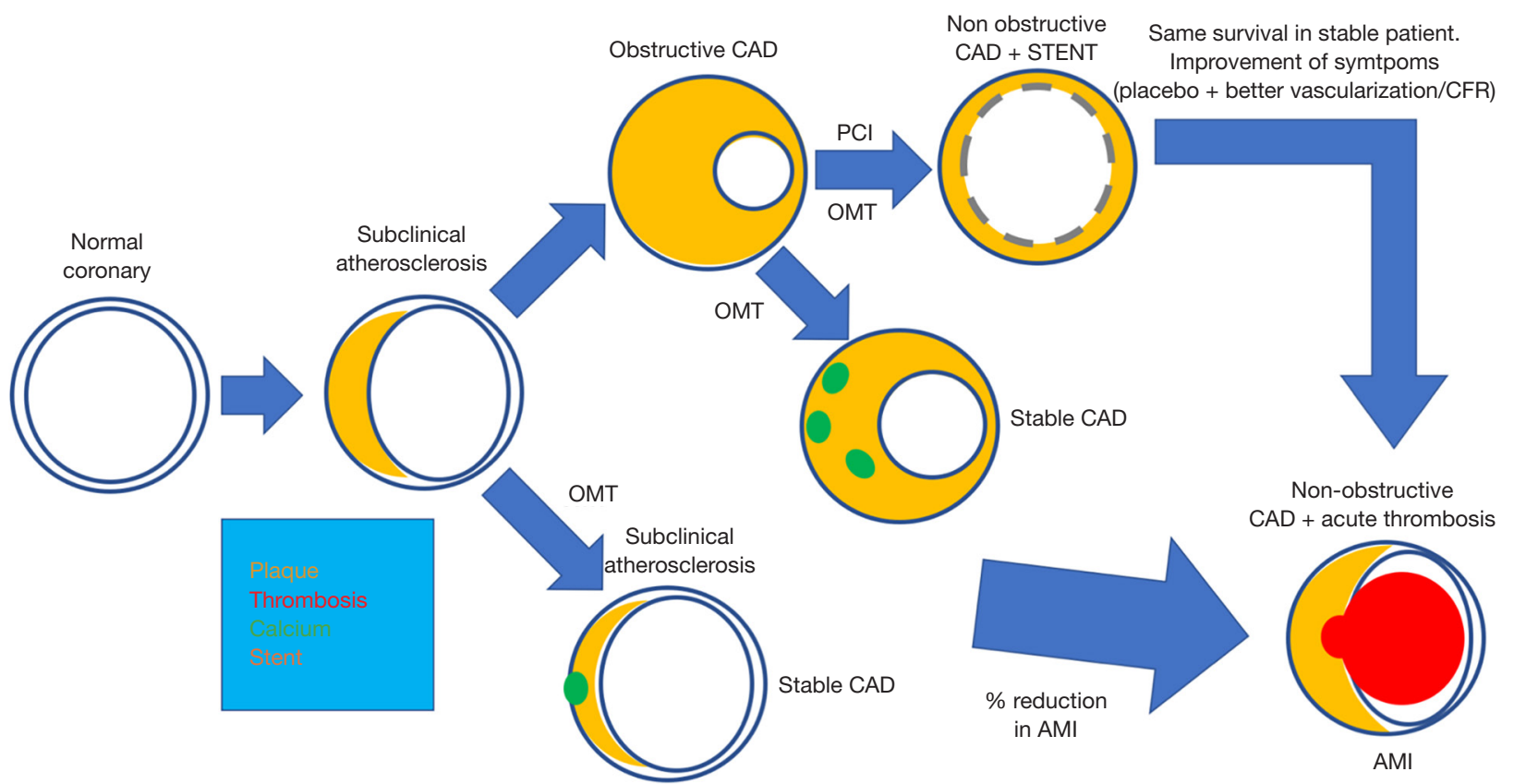

Figure 2 Optimal medical therapy vs. percutaneous coronary intervention. The figure shows a simplified version of natural history of CAD with the two paths of OMT and PCI. OMT, optimal medical therapy; PCI, percutaneous coronary intervention; AMI, acute myocardial infarction; CAD, coronary artery disease; cFR, coronary flow reserve.

soon as the probability of specific disease increase, we want to detect, image and possibly quantify/grade the disease. In many cases we want to exclude the actual disease, which works fine as well.

For decades this approach in Cardiology and in particular in the field of coronary heart disease has been always indirect, until coronary imaging was performed with invasive tools [i.e., invasive coronary angiography (CAG)]. There was no other way. For a long time, we have told that looking at atherosclerosis at the level of carotid arteries was a good way to look at the atherosclerosis of coronary arteries. Honestly, this is something difficult to accept even today that this concept has been debunked.

Diagnostic Cardiology was based on signs \& symptoms, Electrocardiogram, treadmill test, echocardiography, stress SPECT, stress CMR, etc., but none of these tests was actually looking at atherosclerosis inside the coronary arteries.

Then, during the late eighties and nineties, electronbeam computed tomography (EBCT) equipment was developed and it became possible to directly see very well coronary calcifications. There was big excitement because it was possible to see also coronary arteries but well because the spatial resolution was too low and images were too noisy; but calcifications were exciting and extensive studies were performed that showed in a nutshell that coronary calcium score (CACS) is a powerful independent predictor of coronary events in asymptomatic population, especially useful in individuals with low-intermediate risk; today this is in the American guidelines on cardiovascular prevention.

At this point, part of the coronary puzzle was available. But it was a too small piece of information.

In the late nineties and beginning of the new millennium a new technology was developed. It was multislice computed tomography (MSCT), which today is just CT. This technology immediately showed the potential to perform coronary angiography like any other non-invasive CT angiography. Several technical issues were progressively solved through the years concerning speed, radiation dose, contrast material, contrast resolution, spatial resolution, and so forth.

In the last decade, the technical development of CCT became steadier while clinical studies actually exploded providing very strong and extensive evidence of the role of the method in clinical practice. Large studies, registries, and ultimately randomized controlled trials contributed greatly 


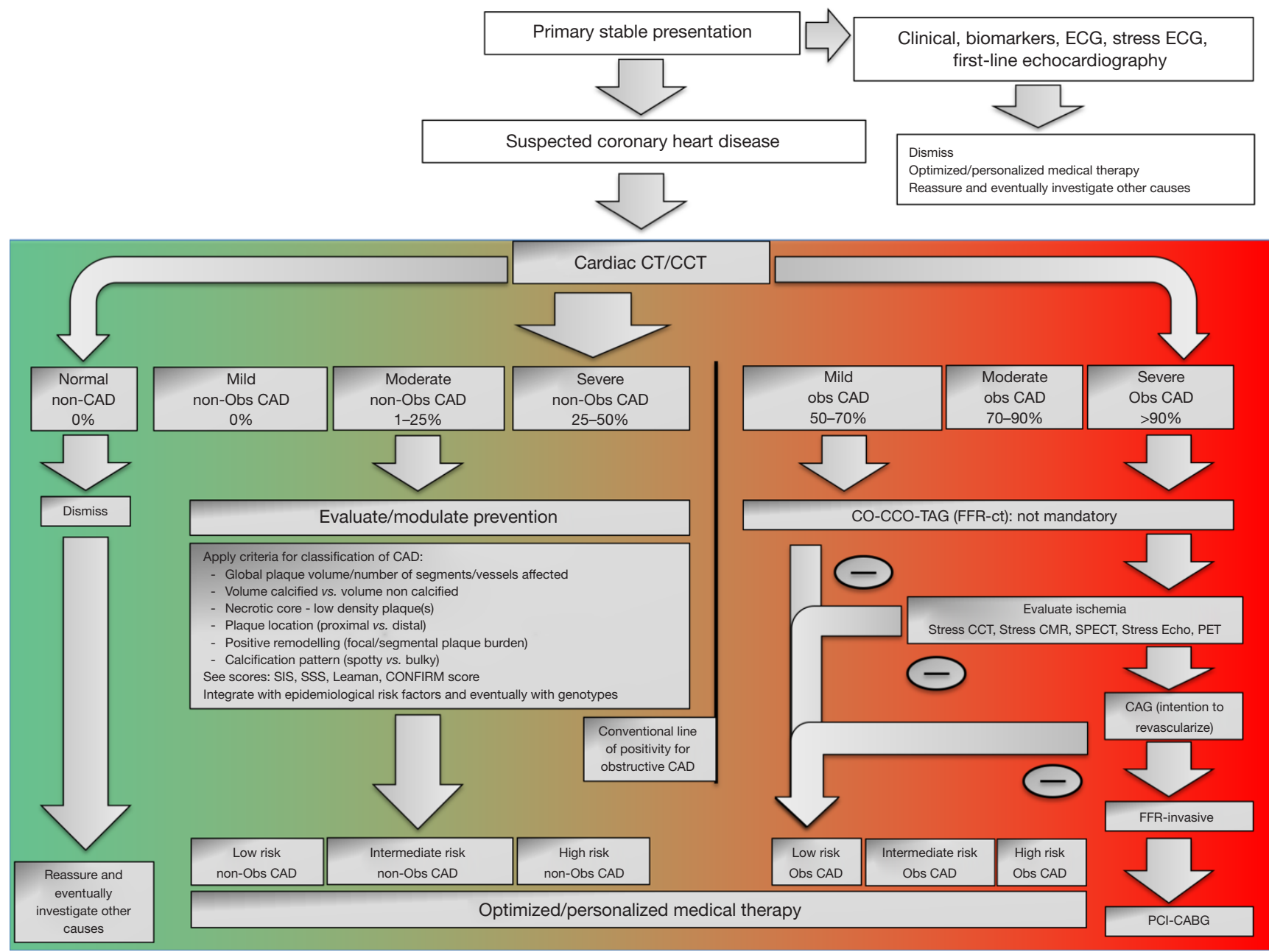

Figure 3 Modern algorithm for suspected coronary artery disease. The figure shows how cardiac CT (CCT) has the primary and pivotal role in defining the CAD phenotype in the first place and off course also the role of defining the degree of obstruction of coronary arteries. While providing this key information, CCT determines also what happens next. For simplicity we divided the panel into two main areas (i.e., a green on the left and red area on the right, representing also the severity of CAD). When we fall on the non-obstructive side of spectrum (left side) the most relevant information are collected in the light of potential future containment of risk factors and preventative therapies; on the other hand, when we fall in the obstructive side of the spectrum (right side), we need to go deeper in the functional impact of CAD. ECG, electrocardiogram; CHD, coronary heart disease; CT, computed tomography; Non Obs, non-obstructive; Obs, obstructive; CAD, coronary artery disease; CO, coronary opacification; CCO, corrected coronary opacification; TAG, transluminal attenuation gradient; CMR, cardiac magnetic resonance; SPECT, single-photon emission computed tomography; Echo, echocardiography; PET, positron emission tomography; CAG, conventional coronary artery angiography; PCI, percutaneous coronary intervention; CABG, coronary artery bypass graft; FFR, fractional flow reserve.

in establishing CCT as a primary clinical tool in suspected obstructive CAD.

\section{The new paradigm (Figure 3)}

What if we had CCT before we had CAG? This is an important question because if affects the way today the Cardiological community still looks at the diagnostic role of CAG. All the prolific growth of indirect and surrogate markers for CAD over the past 50 years has been justified in the fact that CAG was the only method providing direct visualization of coronary arteries. In fact, CAG has several 
limitations too, beyond the fact that it is invasive and therefore not risk-free. It does not show atherosclerosis, it does not show arterial wall remodeling, it does not show the inherent features of atherosclerosis that are quite meaningful nowadays to define what kind of phenotype are we dealing with, and so forth. For these reasons over time, several intravascular imaging techniques have been developed. These techniques are mainly able to fill the gap in qualitative and quantitative evaluation of coronary artery wall [e.g., intravascular ultrasound (IVUS), optical coherence tomography (OCT), etc.]. They proved to be very useful also in the assessment of pre- and postangioplasty/stenting assessment of coronary artery wall and in the assessment of complications of the procedure.

Unfortunately, the implementation of invasive intravascular techniques does not solve the problem of routine coronary imaging. We cannot perform invasive intravascular coronary imaging because of additional cost, risk and ultimately, we can do it when we have a good reason in the context of interventional procedures.

Finally, CCT completed the spectrum of tools. In good hands, CCT can provide all information of a diagnostic CAG and beyond because it is able to show, characterize and quantify plaque burden, beside every anatomical 3-dimensional detail. When we acknowledge the role of CCT we define also a certain level of competence and skills which are related to the technical and interpretational part of the process. It takes a significant amount of time to adequately prepare a CCT team that will be able to deliver the expected quality standards.

What happened in the last 2-3 years goes way beyond the recognition of a reliable diagnostic tool. An alignment of studies, not all strictly related to the role of CCT, brought to the inevitable conclusion that CCT is the primary tool to use.

We will develop the reasoning starting from the latest evidence and going backwards.

At the end of 2019 the European Society of Cardiology presented the new guidelines on chronic coronary syndrome (21); this document finally places CCT in Class I for the assessment of suspected CAD in symptomatic patients. Even if this was expected, it was not at all obvious because there has been strenuous resistance to this recognition for several years, especially from the side functional imagers Cardiologist. Almost at the same time, few weeks later, the preliminary results of the NIH funded ISCHEMIA trial are presented at the American Heart Association 2019; this is the most awaited diagnostic trial of the last decades in which more than

A total of 5,000 patients with stable angina were followed for 3.3 years along 2 randomized arms, one treated with percutaneous coronary intervention (PCI) and one treated with optimal medical therapy (OMT). PCI resulted to be not superior in reducing major cardiovascular events as compared to OMT in stable patients with moderate ischemia. This result is in line the previously published COURAGE study in 2007 and in 2015 with 15 years of follow-up $(23,24)$.

Moreover, already in 2017 the ORBITA study already showed in 230 stable patients with single-vessel obstructive disease $(>70 \%)$ that randomizing them into a sham PCI and a real PCI in both cases with OMT at 6 weeks there was no difference in the exercise capacity between the two arms; this explains the power of placebo effect of PCI.

More recently the controversial result of the EXCEL trial has been presented at TCT 2019; this trial showed no significant difference between the PCI arm and CABG arm in 1,905 randomized patients in terms of survival at 5 years (i.e., death + stroke + AMI 22\% with PCI and 19.2\% with $\mathrm{CABG}$ ). To strengthen these results, a few days later also the results of the NOBLE trial have been published and they showed in 1,201 patients with 5 years follow-up that patients with left main significant disease have better prognosis if treated with CABG as compared to PCI.

Before this recent flood of clinical information, we had mainly two major randomized controlled trial that were specifically designed to verify the role of CCT. The PROMISE trial and the SCOT-HEART trial (29-32). They both have patients with suspected obstructive CAD at the beginning. The PROMISE trial randomized immediately into anatomical arm (CCT) vs. functional arm (standard of care/SOC with functional tests), while the SCOTHEART randomized a standard of care arm (mostly with functional tests) $v s$. the same + CCT; in this case CCT was simply added to standard of care (29-31). The first results of the PROMISE trial showed a substantial equivalence of diagnostic and prognostic performance of the two arms, even though further analyses with longer follow-ups showed a better outlook for the anatomical arm (32). What was really unexpected especially in terms of magnitude was the striking better prognosis of patients in SCOT-HEART in the arm with SOC + CCT. Neither in PROMISE nor in SCOTHEART the protocol included guidelines on how the manage or use the results of tests, letting all decisions in the hands of the referring physicians. In SCOT-HEART, probably for the first time in history of cardiology, the only fact of using 
a test improved significantly the prognosis of patients. This have been further investigated and in seems that the effect is probably attributable to more aggressive medical treatment in patients with non-obstructive CAD and also better medical treatment compliance of these same patients.

The combined effect of all this evidence in a relatively short period of time has changed the paradigm, and it is a clinical paradigm not a theoretical or hypothetical one. The following is a very synthetic list of statements to privilege clarity:

* When there is a significant coronary artery stenosis and the patient is stable, putting a stent does not increase survival; it is optimal medical therapy that plays the most important role;

* Even in patients with significant stenosis of the left main, using stent instead of bypass surgery does not improve survival (it is actually worse);

* It is clear that the role of atherosclerosis imaging is outpacing by far the role functional tests; it is more important to see, assess and quantify atherosclerosis as first step. Functional imaging comes in when ischemia needs to be evaluated; anyway as a second step;

* A patient with suspected obstructive CAD should be referred to CCT as a first step;

- A symptomatic stable patient with no left main significantly obstructive disease at CCT can follow an OMT path which can become a PCI and/or a CABG if OMT is not sufficient;

* The implementation of CCT as primary tool for Chronic Coronary Syndrome is going to positively impact the length and the cost of conventional diagnostic algorithms based on simple tests including functional tests.

There are some things that should be noted. CCT is the solution is most situations. In fact, when we propose CCT as the primary tool we intend that CCT is handled by super-experienced operators in specialistic environments. Without the adequate state-of-art expertise CCT loses most of its advantages (exactly as it happens with CMR).

\section{Newer clinically relevant applications of CCT}

While the major paradigm shift is described and mostly focused on atherosclerosis and plaque imaging and characterization, there also some new CCT techniques that have a great potential in clinical routine and that focus predominantly on the assessment of ischemia. The main applications in this field are CT perfusion (CTP) and fractional flow reserve CT (FFR-CT). With CTP we basically apply the same strategy that we apply for Cardiovascular MR perfusion; we perform dynamic imaging during first pass of contrast material through the myocardium to identify regions/segments of slow/late/absent perfusion, during stress and at rest. With FFR-CT instead we surrogate the pressure drop after a coronary stenosis using the CCT dataset normally acquired for the purpose of anatomical assessment of coronary stenosis; by using accurate vessel segmentation and advanced fluid-dynamics algorithm it is possible to derive an FFR value, in analogy with what is normally performed with an intracoronary catheter. These two applications have the great potential to allow CCT to become a one-stop-shop for all patients with suspected CAD and/or chronic coronary syndrome.

\section{After a new paradigm, do we need new endpoints? (Figure 4)}

Current evidence in terms of diagnostic algorithms and incremental clinical value point at the fact that atherosclerosis is the diagnostic and therapeutic target of modern cardiovascular medicine especially for CAD. Extensive work has already been performed to progressively define the role of different components of atherosclerosis as detected and characterized by CCT (see the CONFIRM, ICONIC and PARADIGM registries). More work is undergoing, and even more will be done in the next decade. Peculiar information can be extrapolated and apparently the most important ones that today seem within reach of clinical implementation are total plaque burden and highrisk plaques identification.

The atherosclerotic plaque features that are more interesting in terms of routine detectability during CCT are: total plaque volume, predominantly non calcified plaque, positive remodeling, napkin ring sign, micro-spotty calcifications, low density plaque core. These features all define a high-risk plaque and the risk is incremental when they are present together.

We need to add to these features some newly discovered parameters with the potential to further improve risk stratification, such as epicardial fat quantification and modification, fractional flow reserve derived methods, and spectral imaging.

The translational nature of this information is the possibility to assess the modifications of the characteristics of atheroma over time, to monitor and eventually quantify 


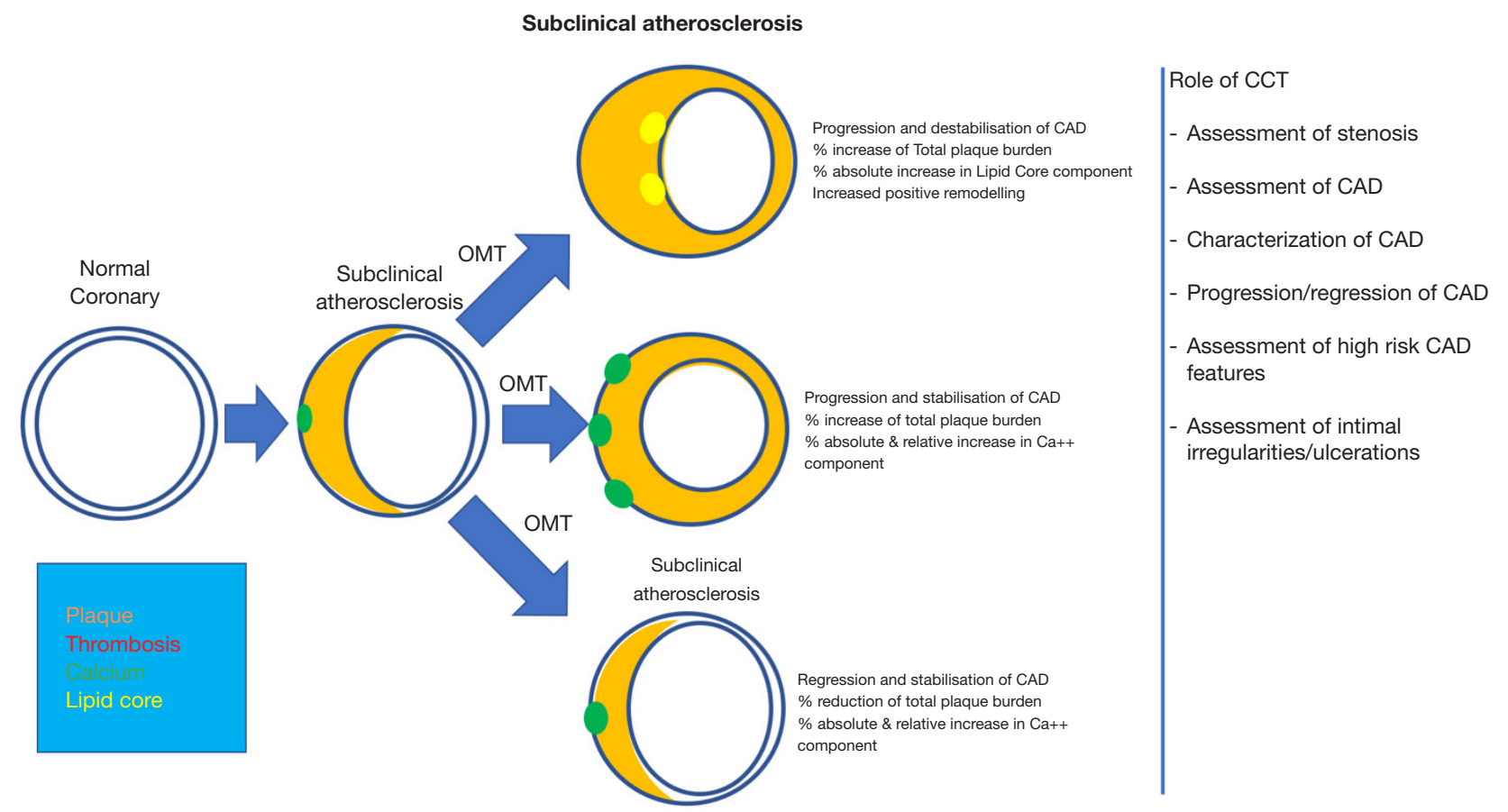

Figure 4 Potential role of CCT in monitoring medical therapy (progression/regression). The Figure shows the potential role CCT in the assessment and monitoring of atherosclerosis natural history during OMT. CCT, cardiac CT; OMT, optimal medical therapy; PCI, percutaneous coronary intervention; AMI, acute myocardial infarction; CAD, coronary artery disease.

the anti-atherogenic effect of current (e.g., statins) and new drugs (e.g., PCSK9), to identify responder from nonresponder to new therapies, and so forth. This whole new field of imaging-driven personalized medical therapy is extremely exciting and with great potential especially in the asymptomatic population.

\section{Conclusions}

A new paradigm of early assessment of atherosclerosis is in place concerning coronary arteries by means of the introduction in routine clinical practice of CCT. This change has several implications one of which is the possibility to address the phenotype of atherosclerosis at very early stages of the natural history of CAD. Therefore, we have to modify our design of research studies and eventually our clinical endpoints during medical and interventional treatment of CAD.

\section{Acknowledgments}

Funding: None.

\section{Footnote}

Provenance and Peer Review: This article was commissioned by the Guest Editor (Filippo Cademartiri) for the series "Clinical Impact of Cardiac CT in Clinical Practice" published in Cardiovascular Diagnosis and Therapy. The article was sent for external peer review organized by the Guest Editor and the editorial office.

Reporting Checklist: The authors have completed the Narrative Review Reporting checklist. Available at http:// dx.doi.org/10.21037/cdt-20-525

Conflicts of Interest: All authors have completed the ICMJE uniform disclosure form (available at http://dx.doi. org/10.21037/cdt-20-525). The series "Clinical Impact of Cardiac CT in Clinical Practice" was commissioned by the editorial office without any funding or sponsorship. FC served as the unpaid Guest Editor of the series and serves as an unpaid editorial board member of Cardiovascular Diagnosis and Therapy from Jul 2019 to Jun 2021. LS serves as an unpaid editorial board member of Cardiovascular Diagnosis and Therapy from Jul 2019 to Jun 2021. The 
authors have no other conflicts of interest to declare.

Ethical Statement: The authors are accountable for all aspects of the work in ensuring that questions related to the accuracy or integrity of any part of the work are appropriately investigated and resolved.

Open Access Statement: This is an Open Access article distributed in accordance with the Creative Commons Attribution-NonCommercial-NoDerivs 4.0 International License (CC BY-NC-ND 4.0), which permits the noncommercial replication and distribution of the article with the strict proviso that no changes or edits are made and the original work is properly cited (including links to both the formal publication through the relevant DOI and the license). See: https://creativecommons.org/licenses/by-nc-nd/4.0/.

\section{References}

1. Nieman K, Cademartiri F, Lemos PA, et al. Reliable noninvasive coronary angiography with fast submillimeter multislice spiral computed tomography. Circulation 2002;106:2051-4.

2. Mollet NR, Cademartiri F, Nieman K, et al. Multislice spiral computed tomography coronary angiography in patients with stable angina pectoris. J Am Coll Cardiol 2004;43:2265-70.

3. Mollet NR, Cademartiri F, van Mieghem CA, et al. High-resolution spiral computed tomography coronary angiography in patients referred for diagnostic conventional coronary angiography. Circulation 2005;112:2318-23.

4. Weustink AC, Mollet NR, Neefjes LA, et al. Diagnostic accuracy and clinical utility of noninvasive testing for coronary artery disease. Ann Intern Med 2010;152:630-9.

5. Maffei E, Palumbo AA, Martini C, et al. "In-house" pharmacological management for computed tomography coronary angiography: heart rate reduction, timing and safety of different drugs used during patient preparation. Eur Radiol 2009;19:2931-40.

6. Cademartiri F, Raaijmakers RH, Kuiper JW, et al. Multidetector row CT angiography in patients with abdominal angina. Radiographics 2004;24:969-84.

7. Migliore F, Maffei E, Perazzolo Marra M, et al. LAD coronary artery myocardial bridging and apical ballooning syndrome. JACC Cardiovasc Imaging 2013;6:32-41.

8. Min JK, Berman DS, Dunning A, et al. All-cause mortality benefit of coronary revascularization vs. medical therapy in patients without known coronary artery disease undergoing coronary computed tomographic angiography: results from CONFIRM (COronary CT Angiography EvaluatioN For Clinical Outcomes: An InteRnational Multicenter Registry). Eur Heart J 2012;33:3088-97.

9. Nakazato R, Gransar H, Berman DS, et al. Statins use and coronary artery plaque composition: results from the International Multicenter CONFIRM Registry. Atherosclerosis 2012;225:148-53.

10. Labounty TM, Gomez MJ, Achenbach S, et al. Body mass index and the prevalence, severity, and risk of coronary artery disease: an international multicentre study of 13,874 patients. Eur Heart J Cardiovasc Imaging 2013;14:456-63.

11. Cho I, Chang HJ, Sung JM, et al. Coronary computed tomographic angiography and risk of all-cause mortality and nonfatal myocardial infarction in subjects without chest pain syndrome from the CONFIRM Registry (coronary CT angiography evaluation for clinical outcomes: an international multicenter registry). Circulation 2012;126:304-13.

12. Small GR, Yam Y, Chen L, et al. Prognostic assessment of coronary artery bypass patients with 64-slice computed tomography angiography: anatomical information is incremental to clinical risk prediction. J Am Coll Cardiol 2011;58:2389-95.

13. Villines TC, Hulten EA, Shaw LJ, et al. Prevalence and severity of coronary artery disease and adverse events among symptomatic patients with coronary artery calcification scores of zero undergoing coronary computed tomography angiography: results from the CONFIRM (Coronary CT Angiography Evaluation for Clinical Outcomes: An International Multicenter) registry. J Am Coll Cardiol 2011;58:2533-40.

14. Cheng VY, Berman DS, Rozanski A, et al. Performance of the traditional age, sex, and angina typicalitybased approach for estimating pretest probability of angiographically significant coronary artery disease in patients undergoing coronary computed tomographic angiography: results from the multinational coronary CT angiography evaluation for clinical outcomes: an international multicenter registry (CONFIRM). Circulation 2011;124:2423-32, 1-8.

15. Min JK, Dunning A, Lin FY, et al. Age- and sex-related differences in all-cause mortality risk based on coronary computed tomography angiography findings results from the International Multicenter CONFIRM (Coronary CT Angiography Evaluation for Clinical Outcomes: An International Multicenter Registry) of 23,854 patients 
without known coronary artery disease. J Am Coll Cardiol 2011;58:849-60.

16. Chow BJ, Small G, Yam Y, et al. Incremental prognostic value of cardiac computed tomography in coronary artery disease using CONFIRM: COroNary computed tomography angiography evaluation for clinical outcomes: an InteRnational Multicenter registry. Circ Cardiovasc Imaging 2011;4:463-72.

17. Cademartiri F, La Grutta L, Palumbo AA, et al. Coronary plaque imaging with multislice computed tomography: technique and clinical applications. Eur Radiol 2006;16 Suppl 7:M44-53.

18. Cademartiri F, La Grutta L, Palumbo A, et al. Imaging techniques for the vulnerable coronary plaque. Radiol Med 2007;112:637-59.

19. Cademartiri F, Schuijf JD, Pugliese F, et al. Usefulness of 64-slice multislice computed tomography coronary angiography to assess in-stent restenosis. J Am Coll Cardiol 2007;49:2204-10.

20. Cademartiri F, La Grutta L, Runza G, et al. Influence of convolution filtering on coronary plaque attenuation values: observations in an ex vivo model of multislice computed tomography coronary angiography. Eur Radiol 2007;17:1842-9.

21. Knuuti J, Wijns W, Saraste A, et al. ESC Scientific Document Group 2019 ESC Guidelines for the diagnosis and management of chronic coronary syndromes. Eur Heart J 2020;41:407-77.

22. Available online: https://www.acc.org/latest-in-cardiology/ clinical-trials/2019/11/15/17/27/ischemia

23. Boden WE, O'Rourke RA, Teo KK, et al. Optimal medical therapy with or without PCI for stable coronary disease. $\mathrm{N}$
Engl J Med 2007;356:1503-16.

24. Sedlis SP, Hartigan PM, Teo KK, et al. Effect of PCI on Long-Term Survival in Patients with Stable Ischemic Heart Disease. N Engl J Med 2015;373:1937-46.

25. Stone GW, Kappetein AP, Sabik JF, et al. Five-Year Outcomes after PCI or CABG for Left Main Coronary Disease. N Engl J Med 2019;381:1820-30.

26. Available online: https://www.tctmd.com/news/excel-5years-pci-left-main-cad-still-noninferior-cabg

27. Holm NR, Mäkikallio T, Lindsay MM, et al. Percutaneous coronary angioplasty versus coronary artery bypass grafting in the treatment of unprotected left main stenosis: updated 5-year outcomes from the randomised, noninferiority NOBLE trial. Lancet 2020;395:191-9.

28. Neglia D, Rovai D, Caselli C, et al. Detection of significant coronary artery disease by noninvasive anatomical and functional imaging. Circ Cardiovasc Imaging 2015;8:e02179.

29. Williams MC, Moss AJ, Dweck M, et al. Coronary Artery Plaque Characteristics Associated With Adverse Outcomes in the SCOT-HEART Study. J Am Coll Cardiol 2019;73:291-301.

30. Williams MC, Adamson PD, Newby DE. Coronary CT Angiography and Subsequent Risk of Myocardial Infarction. N Engl J Med 2019;380:300.

31. SCOT-HEART Investigators, Newby DE, Adamson PD, et al. Coronary CT Angiography and 5-Year Risk of Myocardial Infarction. N Engl J Med 2018;379:924-33.

32. Douglas PS, Hoffmann U, Patel MR, et al. Outcomes of anatomical versus functional testing for coronary artery disease. N Engl J Med 2015;372:1291-300.
Cite this article as: Maffei E, Punzo B, Cavaliere C, Bossone E, Saba L, Cademartiri F. Coronary atherosclerosis as the main endpoint of non-invasive imaging in cardiology: a narrative review. Cardiovasc Diagn Ther 2020;10(6):1897-1905. doi: $10.21037 / \mathrm{cdt}-20-525$ 